\title{
THE CHEMICAL CHARACTERIZATION AND PROCESSING SCIENCE OF COMPOSITES
}

\author{
Clayton A. May \\ Lockheed Missiles \& Space Company, Inc. \\ Sunnyvale, California
}

\begin{abstract}
To completely control the process of fabricating composite hardware, it is necessary to assure the starting prepreg is chemically consistent and that all the chemical and physical changes of the process have occurred within the proper time/temperature window. Methods which can be used to control the starting material are discussed. Dielectric monitoring of the cure and a scientific understanding of the processing are considered as a potential key to automated cure cycle control based on realtime chemical and physical matrix parameters.
\end{abstract}

Because of favorable strength to weight ratios, fiber reinforced composite materials are gradually replacing metal structures in the aerospace industry. Conventional metal structures are fabricated by mechanical processes. Composites, however, are generally formed by the hardening of an organic matrix polymer formulation in which fibers have been embedded in a predetermined geometric pattern. Fabrication of composite hardware thus must be considered a chemical process. The responsibility for the fabrication accordingly must shift from the mechanical engineer to the chemical engineer.

In chemical processes, reliable products are the logical result of using chemically well defined raw materials. The processes are controlled and documented by monitoring the occurrence of chemical and physical changes. With these data available for composites, part quality could be assured by. non-destructive evaluation methods which verified that the structure is sound and free from major or excessive small void content.

Until about ten years ago quantitative and qualitative identification of the starting reactants in thermosetting resin formulations was a difficult and time consuming process. With the recent advances in sophisticated instrumentation, chemical identification schemes have become easier and more cost effective. Quantitative identification of the proper chemical formulation, including B-stage or advancement can be accomplished in a few hours as compared to the approximately 40 hour effort needed to identify that a purchased product (prepreg) would meet a series of short time mechanical requirements. Further, these latter tests do not identify chemical changes in a matrix formulation which could impact long term mechanical performance. This is an important consideration realizing that human lives and costly products could be lost due to unknown time dependent failures.

Concurrent with the development of formulation analysis methods, work in our own laboratories showed that changes in the dielectric properties of a curing thermosetting resin presented a dynamic picture of the chemical and physical events which occur during the processing of composite structures. Thus a means of controlling and monitoring the cure process was also available. In truth, the title of this talk is a redundancy. Hithout knowing the chemical structure and concentration of the reactants it becomes impossible to delineate the science of composite processing. Conversely, without a process monitoring system, there can be no assurance that a desired formulation has properly reacted.

The development of a scientifically controlled composite hardware fabrication process is not an easy task. It results only through a multi-disciplined scientific approach. Technological skills are required in the fields of rheology, reaction kinetics, microelectronics, mathematics, tooling, heat transfer, predictive modeling, spectroscopy, reaction mechanisms, thermal analysis, dielectric analysis, chromatography, computer science and programming, adhesion, resin chemistry and formulation and analytical chemistry. A team must thus be built consisting of analytical, physical and polymer chemists, spectroscopists, chemical, electronics and manufacturing engineers and computer skilled mathematicians. It is the purpose of this paper to review the current state-of-the-art in the chemical cilaracterization of matrix materials and show that dielectric analysis can be used to develop automated cure cycle control. Using the latter approach it is possible to control composite curing processes using 
computer based monitoring of the process chemistry and rheology. Thus all process decisions such as the control of heating rates and pressure application points are freed from the inherent errors of human judgement.

The resin systems of current vogue in the aerospace industry today are largely based on glycidyl amine formulations involving tetraglycidylmethylenedianiline (TGMDA) I and diaminodiphenylsulfone (DDS) II. These raw materials are commercially available in Asia, Europe and<smiles>c1cc(CN(CC2CO2)CC2CO2)ccc1CCN1CCC(CC2CO2)C1</smiles>

Tetraglycidylmethylenedianiline (TGMDA) I<smiles>Nc1ccc(S(=O)(=O)c2ccccc2)cc1</smiles>

Diaminodiphenylsulfone (DDS) II

the United States. Composite prepreg formulations containing these products also contain auxiliary catalysts, secondary resins and diluents, complicating the chemical and physical characterization of the composite starting material. The first part of this presentation reviews the current status of chemical characterization methodology including some physical characterization procedures. These latter tecinniques have been found to be highly important in that predictable behavioral characteristics in a laminating raw material is of logical and equal importance to its chemical composition.

A number of characterization techniques have evolved over the past few years which permit the composite fabricator to verify the starting chemical compositions of prepregs and quantize their lamination process behavior. Differential scanning calorimetry defines the onset of the cure, indicates the presence of troublesome volatiles in the prepreg and measures the total heat of reaction of the cure. Chromatography is the heart of chemical composition analysis. It quantitatively defines and identifies the ratios of the unreacted formulation components and permits an estimation of the extent of B-staging. It is an extremely powerful identification tool. In addition to structural verification, infrared can supply valuable quantitative information on formulative components. In the case of a TGMDA/DDS formulation for instance, the total sulfone group concentration can be quantified giving the total amount of DDS in the mixture, both the unreacted material and that which has already reacted with the resin during the B-staging or prepreging process. Wet chemistry methods are traditional and have for many years been used to quantitatively estimate functional group contents such as amines or epoxides. Rheological measurement is a relative newcomer to the characterization field, but none-the-less a highly important one. In the liquid state it can be used to measure how the viscosity or flow characteristics of a given matrix resin or prepreg will change for a given process. In the solid state it can be used to measure post cured properties such as glass transition temperature or modulus as a function of temperature. Dielectric analysis is also a process control tool. In a given process it defines where a matrix starts to flow, the regions of minimum viscosity and the onset of gelation or hardening. These points will be discussed in further detail later in the presentation.

The technological impacts of chemical and physical characterization are many. The prepreg manufacturer is forced to supply consistent raw materials which enhance confidence in predictable hardware and reduce the fabrication of scrap. Without these tools scientifically based lamination process development is a virtual impossibility and it would not be possible to verify that a hardware construction had been properly processed.

Shown in Fig. 1 is a high pressure liquid chromatogram (HPLC) of a typical TGMDA/DDS formulation. Peaks which define the resin, curing agent, diluent and reacted material are clearly evident. The areas under these peaks are directly proportional to the amount of each component present. As a prepreg of this type ages under ambient conditions, not unexpectedly, it slowly reacts. This causes the areas under the peaks to change. 


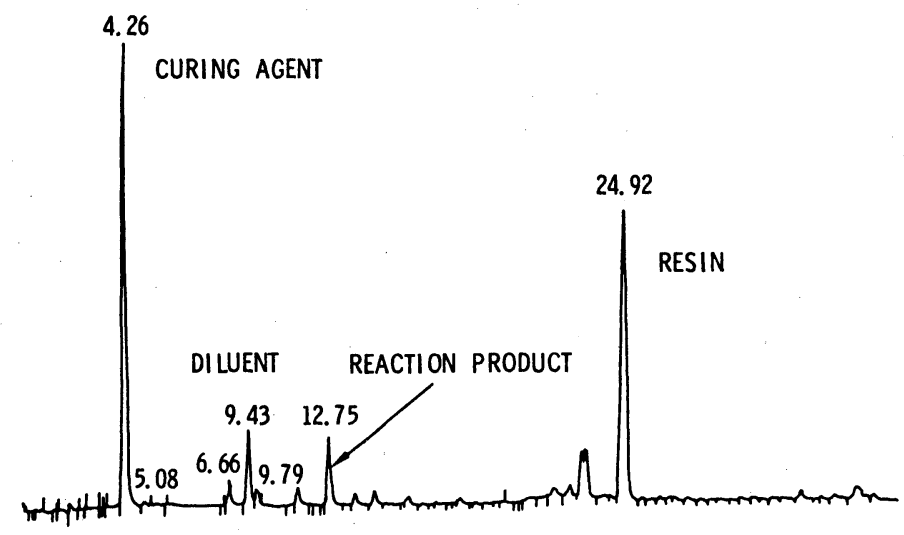

Fig. 1 Chromatogram of a TGMDA/DDS formulation

A summary of an aging experiment is shown in Table 1. It should be noted that as the mater-

TABLE 1. HPLC analysis of an aging process

\begin{tabular}{ccccc} 
Days Aging & \%DDS & \%Diluent & \%TGMDA & \%B-Stage \\
\hline 0 & 30.3 & 13.0 & & \\
2 & 30.1 & 12.8 & 50.0 & 5.7 \\
7 & 29.1 & 12.9 & 46.2 & 6.8 \\
14 & 27.4 & 11.6 & 44.9 & 11.8 \\
30 & 25.3 & 10.3 & 43.1 & 16.1 \\
63 & 22.4 & 9.9 & 43.7 & 24.2
\end{tabular}

ial ages the percentages of the curing agent (DDS), diluent and principal resin (TGMDA) gradually decline while the percentage of B-staged material increases. During the aging process a prepreg material gradually loses its tack and becomes more boardy or glassy in nature. In the glassy state the reactivity or molecular mobility of the reactants is reduced. This is a long established principle 'in polymer chemistry and as the data shows, the aging process slows considerably during the last 30 days of the experiment.

Differential scanning calorimetry monitors the chemical kinetics of a matrix resin formulation. It can reveal very minor changes in a formulation, particularly when minor changes are made in auxiliary catalyst concentrations. Typically, in the TGMDA formulations, salts of Lewis acids and bases are used for this purpose. The effect of catalyst concentration is illustrated by thermograms shown in Fig. 2 wherein a small change in concentration definitely

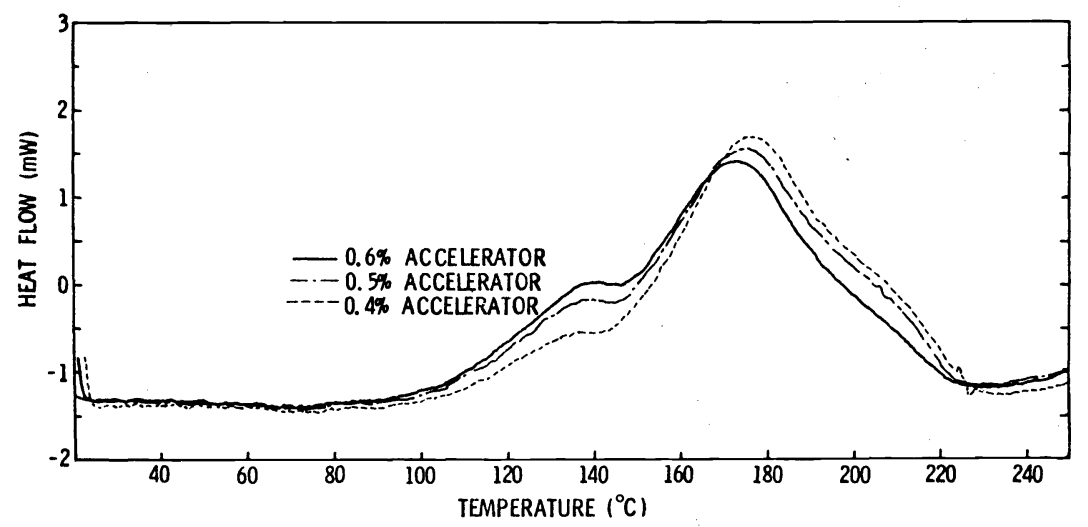

Fig. 2 Influence of catalyst concentration on DSC thermograms

alters the heat flow response of the formulation. The first peak in the heat flow curve has been assigned to the accelerator, the second to the resin/curing agent reactions. As the catalyst concentration increases, the first peak increases and the second peak declines. Since the total epoxide concentration of each formulation is constant, however, the total area under the curves is essentially the same. 
Whereas the changes in the thermogram appear to be of a minor but detectable nature, the impact on processibility can be drastic. This is illustrated by the viscosity data shown in Fig. 3. The logarithm of the viscosity of the three formulations has been plotted as a

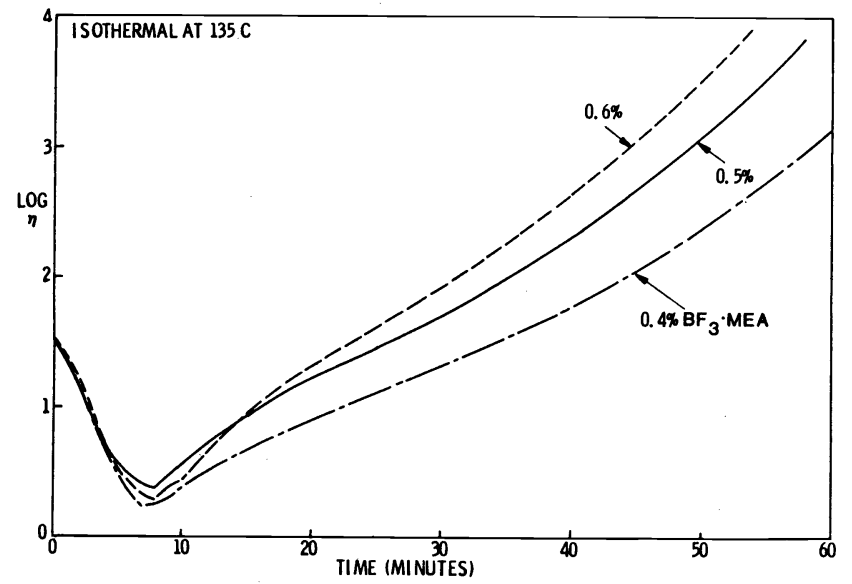

Fig. 3 Effect of accelerator concentration of viscosity

function of time for an isothermal cure at $135^{\circ} \mathrm{C}$. If previous experimentation had shown, for instance, that 1000 poise was the ideal viscosity for lamination consolidation through the application of pressure, this point could vary by 20 minutes on the time scale. The end result could be faulty hardware due to insufficient or excessive resin squeeze out. This clearly indicates the importance of rheological measurements as a prepreg quality assurance procedure.

The value of infrared spectroscopy as a quantitative quality assurance tool has previosuly been discussed. It has also been established that through the use of Fourier Transform (FTIR) techniques the precision of the method can be greatly enhanced particularly when coupled with current computer technologies. An illustration of this point is given in Fig. 4 , the subtractive analysis of an epoxy resin/polyamide mixture. Shown in the upper left
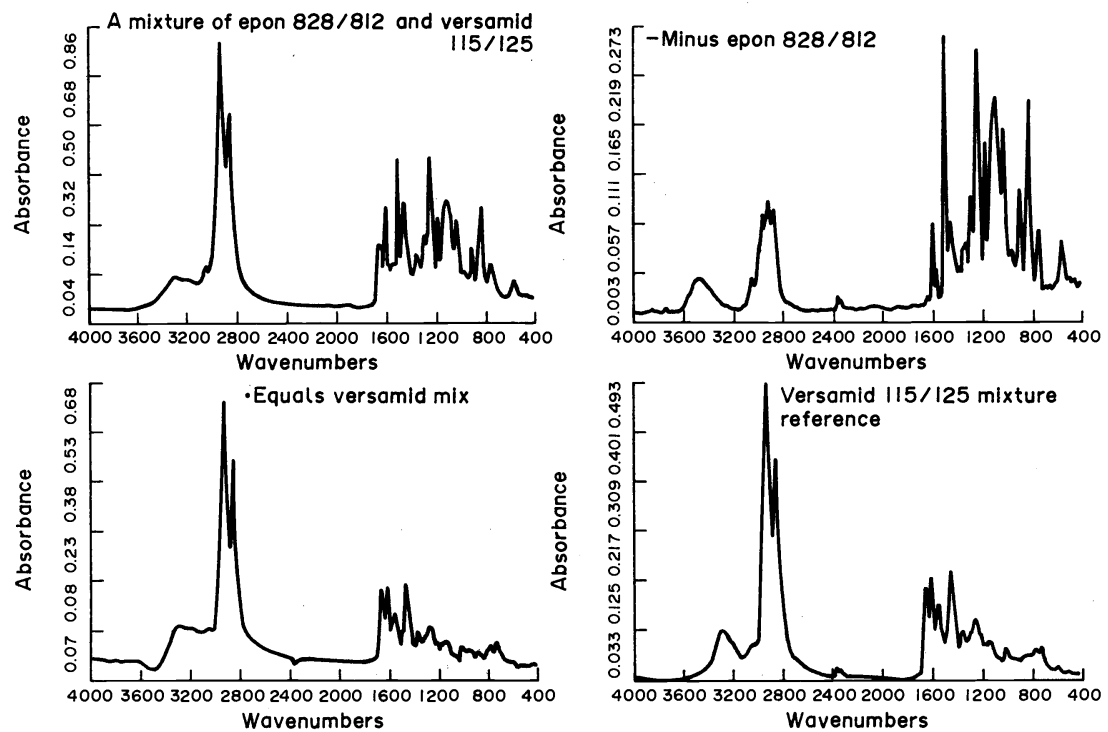

Fig. 4 FTIR subtractive analysis 
hand corner of the figure is the infrared spectrum of the mixture in question. The upper right hand spectrum is that of the resin system prior to the mixing of the ingredients. If the upper right hand spectrum is computer subtracted from that of the mixture on the left, the result is the spectrum in the lower left hand corner of the figure. Note the excellent agreement with the spectrum at the lower right, that of the polyamide curing agent. As our abilities to handle procedures such as this improve, quality assurance procedures will continue to improve in ease and accuracy.

The remainder of this article will be concerned with automatic process control. During the 1970 's our publications demonstrated the applicability of dielectric measurement both as a quality assurance and monitoring tool (1-5). Previous references in the literature indicated the viability of this approach (6-12). It is a well established fact that mechanical and electrical forces are analogus as illustrated by the similarity of their mathematical relationships. A comparison of this type is presented in Table 2 (13). Considering lamin-

TABLE 2. Conversion for force-voltage analogy

\begin{tabular}{ll} 
Mechanical System & Electrical System \\
\hline Force & Voltage \\
Velocity & Current \\
Displacement & Charge \\
Mass & Inductance \\
Damping Coefficient & Resistance \\
Compliance & Capacitance \\
Mechanical Loss & Loss Tangent \\
\hline
\end{tabular}

ation prepreg materials, voltage and current can be regarded as a monitor of viscosity; resistance, capacitance or loss tangent can be related to chemical change since they result from segmental molecular motions. The measurement of dielectric properties for the purpose of process control can be made by a variety of different ways. One approach is illustrated in Fig. 5 which qualitatively compares viscosity and dielectric changes as a function of a simple cure cycle for a composite lamination process.

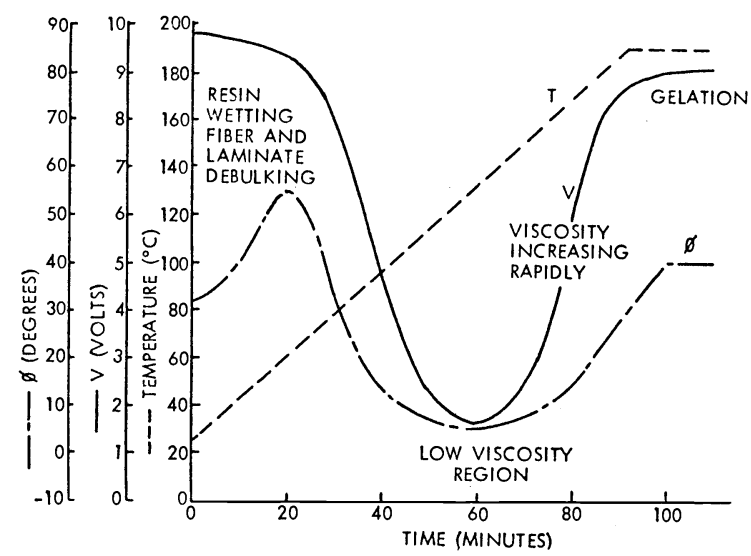

Fig. 5 Influence of physical changes on dielectric properties

In this figure phase angle $(\phi)$, vector voltage $(V)$ and temperature $\left({ }^{\circ} \mathrm{C}\right)$ are plotted as a function of time in an AC field of $1000 \mathrm{~Hz}$. As fiber wetting by the resin and laminate debulking occur, both the phase angle and vector voltage decrease indicating a loosening of the resin matrix molecular structure. The dielectric properties approach a minimum as the resin moves towards minimum viscosity. Subsequently, as the process of gelation and hardening of the matrix sets in molecular motion becomes increasingly restricted resulting in a sharp increase in both phase angle and vector voltage. From these data it is apparent that dielectric data is a real time monitor of physical and chemical changes in a composite lamination process.

As mentioned above, dielectric measurements can be made in a variety of ways. This is i11ustrated in Fig. 6 which is the cure of an elastomer modified epoxy adhesive using a simple 


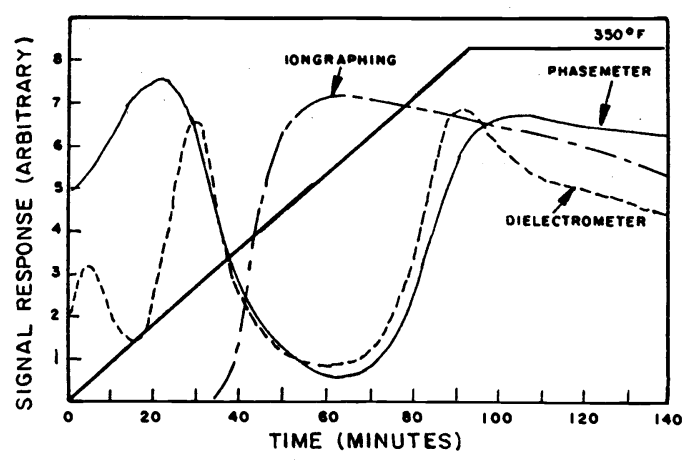

Fig. 6 Comparison of dielectric monitoring traces

cure cycle involving a one hour up heat to $350^{\circ} \mathrm{F}\left(177^{\circ} \mathrm{C}\right)$. Three types of monitoring traces were made; ion graphing which is a simple DC resistivity measurement, loss tangent data obtained from a commercial dielectrometer and the previously mentioned phase angle approach. All three curves reflect the fact that chemical and/or physical changes are occurring as the adhesive cures. We use phase angle and vector voltage measurements for the bulk of our current efforts because of the ready adaptability of the instrumentation to production environments. The $D C$ ion graphing approach has the inherent disadvantage of polarizability of the measurement cell. The commercial dielectrometer is highly sensitive to chemical and physical changes but at times has presented problems in shop environments. We have, however, found this to be an excellent laboratory tool. Note the third peak in the dielectrometer data. This occurs at a temperature where secondary transitions have been observed for the type of elastomer used to modify the adhesive.

At this point in the dissertation a word of caution should be injected concerning formulative change. Earlier studies in our laboratories clearly demonstrated that even subtle changes in the matrix chemical structure cause major variations in the dielectric response during cure (14). A clear illustration of this point is presented from the data in Table 3 and Figs. 7 and 8.

TABLE 3. TGMDA/DDS Matrix formulation

\begin{tabular}{lccc} 
Formula No. & 1 & 2 & 3 \\
\hline TGMDA & 100 & 100 & 100 \\
DDS & 32 & 32 & 32 \\
BF3.400 & - & 1 & - \\
RC-2 & - & - & 1.43 \\
\hline
\end{tabular}

In Table 3 are shown three TGMDA/DDS formulations. All three formulations contain the same resin/curing agent combinations. However, two of the three combinations contain different proprietary accelerators in equivalent active concentrations. This results in different dielectric responses which can also be readily detected by DSC thermograms.

Shown in Fig. 7 are the thermograms of the three formulations as a function of time for a simple composite cure cycle. The important aspects of these data are the cure onset, the shapes of the curves and the areas under the curves. Precursory examination indicates the area under the curves is similar reflecting the fact that the basic resin:curing agent ratios are similar. The differences observed are in the magnitude of the accelerator peaks and the fact that the accelerated systems attain complete cure more rapidly.

These data are reflected in dielectric measurements indicating the importance of recognizing formulative differences through physiochemical examination of starting composite raw materials. Dielectric analysis of the three systems is shown in Fig. 8 and it reflects the DSC data. The accelerator in Formula 3 provides for more rapid cure than that in Formula 2 . Both cure more rapidly than the unaccelerated system. The other point apparent from these data that the time where pressure should be applied to laminate for proper laminate consolidation should be sooner for formula 3 than Formula 2. Thus, dielectric measurement during cure also suggests that it can deal with formulative change in controlling a cure cycle. 


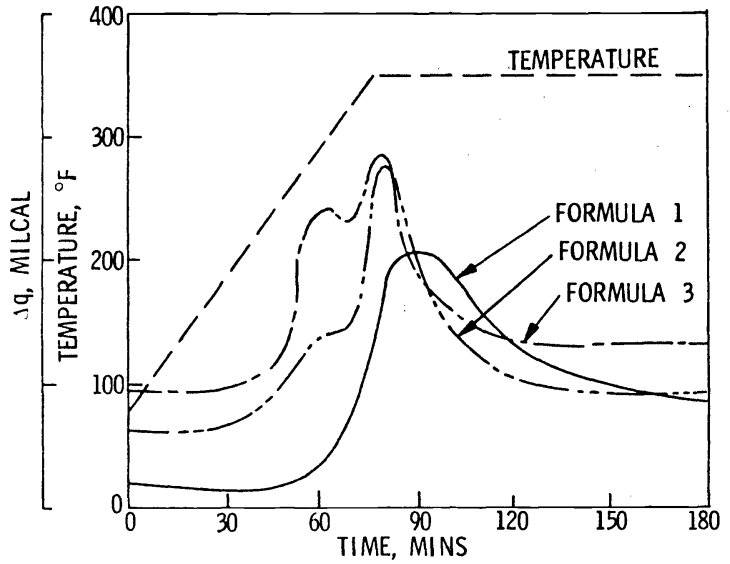

Fig. 7 DSC analysis of TGMDA/DDS formulations

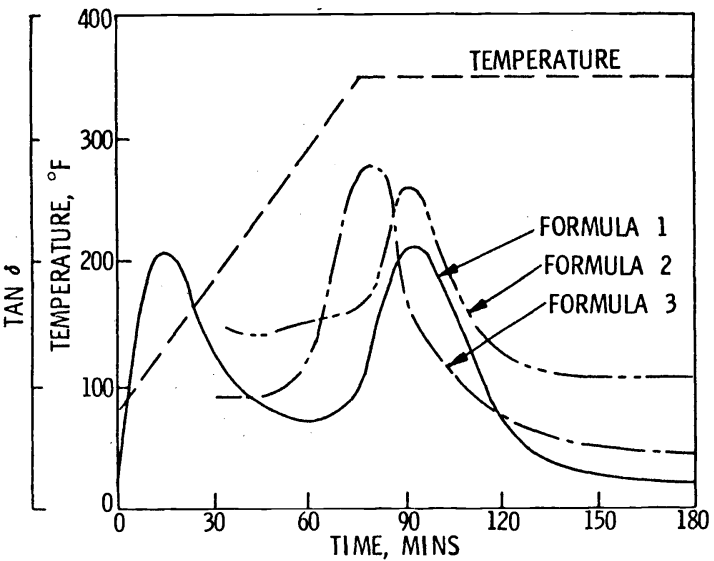

Fig. 8 Dielectric analysis of TGMDA/DDS formulations

There is also a good correlation that can be demonstrated between dielectric analysis data and the changes in viscosity of a curing composite matrix resin. The plot shown in Fig. 9 compares phase angle, vector voltage and matrix viscosity for a stepped cure cycle, typical of many current lamination processes, as a function of process time. The vector voltage (VV) and viscosity curves appear quite similar. In fact the digitized mathematical correlation coefficient between the two measurements is in the range of 90 percent. It should also be noted that minimum viscosity points in each step of the cure cycle correspond to a maximum in the phase angle shift.

These data lead to the conclusion that because of this high correlation between vector voltage and viscosity, it would be possible to evolve an operator independent, closed-loop cure cycle based only on physical or vector voltage change. All that would be required is the development of the proper mathematical algorithm between the two data files. This proved erroneous. Our attempts in this direction were apparently at fault because we had considered only physics of the lamination process and neglected the chemistry.

Our preliminary work has shown that if a mathematical algorithm is developed from both phase angle and vector voltage data it is possible to use dielectric signals to control a lamination process. This means that composite fabrication can be based solely on the chemistry and physics of the curing process and independent of any operator decisions. The result of our preliminary efforts are summarized in Fig. 10.

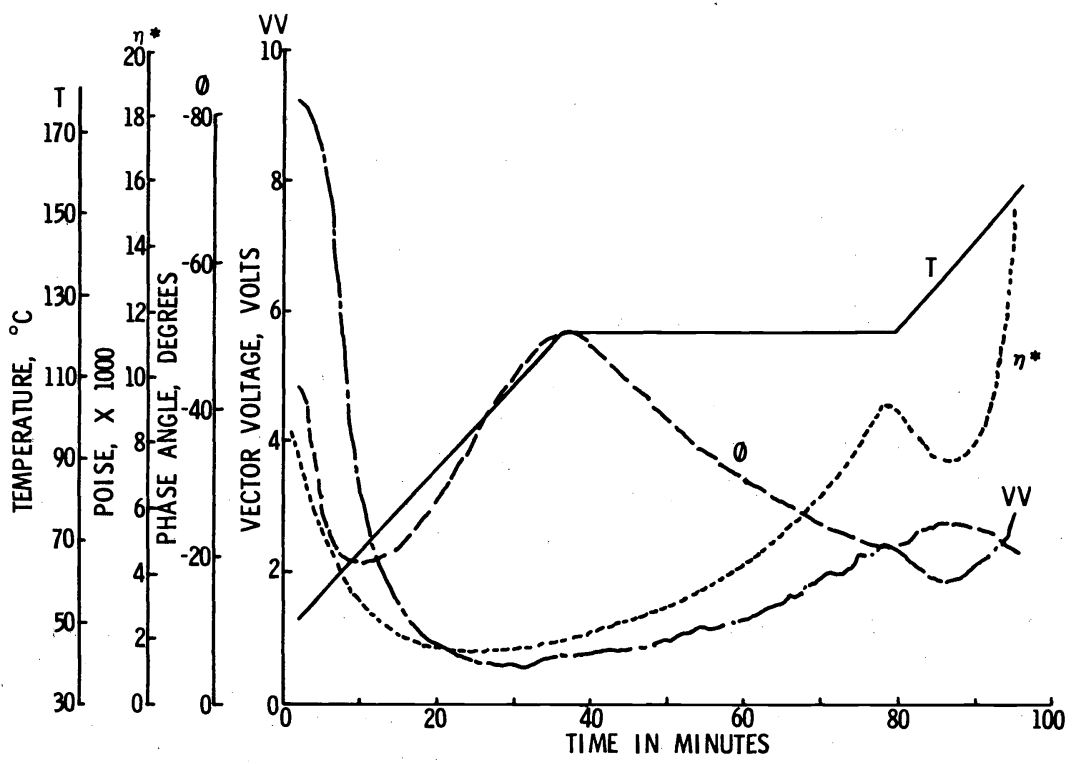

Fig. 9 Comparison of dielectric signals and viscosity 


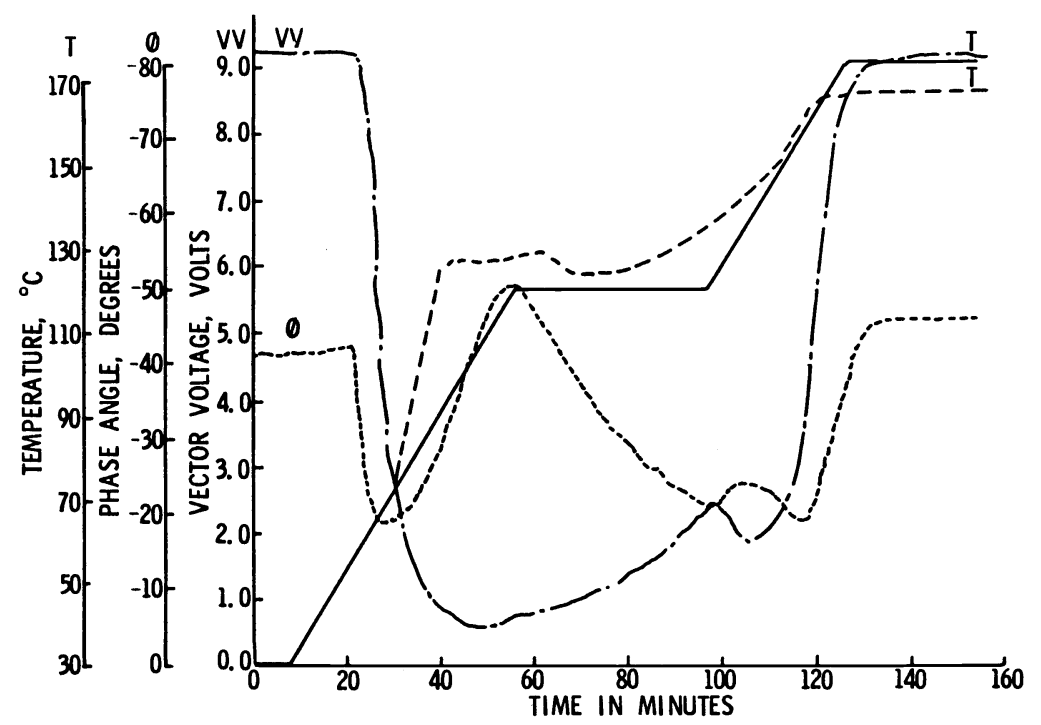

Fig. 10 Normal cure cycle vs. preliminary closed-10op cure cycle

In this figure the baseline cure cycle, the phase angle and the vector voltage used to derive the cure control algorithm are plotted as a function of time. The computer derived cure cycle which resulted from using the dielectric signals to control the temperature is shown by the dashed line. Although it is not an exact replica of the baseline cure cycle the two time-temperature curves are strikingly similar. Since the control algorithm was based on a single run in this case it strongly suggests that additional baseline runs will result in an even closer match between the control and baseline cycles. The success of the method is further attested to by the fact that a composite test panel made using dielectric closed-10op process controls was as good as one prepared using the proven baseline cure cycle.

In conclusion, the data presented herein described how the chemical composition of prepreg resin matrices can be quantitatively measured and controlled through physical and chemical analytical techniques. Fourier Transform Infrared Spectroscopy has great promise as a future tool to be used for this purpose. It has also been shown that composite processing can be controlled in a logical and scientific manner using dielectric responses to monitor the chemical and rheological changes as the cure process proceeds. The current vogue in composite matrix research is to find tougher, more moisture resistant matrices with better high temperature capabilities. As these new products become available, real life cure cycle control through the use of dielectric analytical techniques is realizable. With automated layup procedures, the entire composite hardware fabrication process from the moment the prepreg precuror arrives at factory until the finished part leaves the door can now be done completely free from the errors of human decision.

\section{REFERENCES}

1. C. A. May, J. S. Fritzen and G. R. Brown, International Conference on Composite Materials Proceedings, 264-84 Boston/Geneva (1975).

2. C. A. May, SAMPE Series 20; 108-16 San Diego (Apri1 1975).

3. A. Wereta, Jr., and C. A. May, Org. Coatings and Plastics Chem., ACS Preprints 38, 679-84 Anaheim (March 1978).

4. A. Wereta, Jr., and C. A. May, J. Adhesion 12, 317-331 (1981).

5. J. S. Fritzen, A. Hereta, Jr., and E. A. Arvay, SAMPE Series 22, 430-34 San Diego. (Apri1 1977).

6. A. von Hippel, Dielectric Properties of Materials, MIT Press Cambridge, MA (1954).

7. A. Turner, Jr., Mechanical Behavior of High Polymers, Interscience Publishers New York (1948).

8. C. P. Smyth, Dielectric Behavior and Structure, McGraw-Hill New York (1955).

9. N. G. McCrum, B. E. Reed and G. Williams, Anelastic and Dielectric Effects in Polymeric Solids, John !li ley and Sons New York (1967).

10. W. J. Wrasidlo, Motions in Poly-Pyrometllitimide, Boeing Scientific Research Laboratories Rpt. No. D1-82-1061.

11. S. A. Yaloff and W. J. Wrasidlo, J. Appl. Polymer Sci 16, 2159 (1972).

12. P. Hedvig, Dielectric Spectroscopy of Polymers, John WiTey and Sons New York (1975).

13. D. K. Cheng, Analysis of Linear Systems, Addison-Wesly Publishing Co., Inc. Reading, MA (1961)

14. C. A. May, D. K. Whearty and J. S. Fritzen, SAMPE Series 21, 803-18, Los Angeles (Apri1 $(1976)$. 